Sports Science

\title{
Home-based high-intensity interval training can improve physical performance in young female athletes during a quarantine
}

\author{
Camila Borges Müller $^{1}$ (D), Rousseau Silva da Veiga ${ }^{1}$ (D), Eraldo dos Santos Pinheiro ${ }^{1}$ (D), \\ Fabrício Boscolo Del Vecchio ${ }^{1}$ \\ ${ }^{1}$ Universidade Federal de Pelotas, Escola Superior de Educação Física, Pelotas, RS, Brazil. \\ Associate Editor: Angelina Zanesco (1D), Universidade Metropolitana de Santos, Faculdade de \\ Medicina, Santos, SP, Brazil; Universidade Estadual Paulista "Júlio de Mesquita Filho", \\ Departamento de Educação Física, Instituto de Biociências, Rio Claro, SP, Brazil. E-mail: \\ angelina.zanesco@unesp.br.
}

\begin{abstract}
Aim: To verify the effects of a 2-week of high-intensity interval training (HIIT) on physical performance in young female athletes. Methods: female rugby players $(n=13 ; 15.92 \pm 0.76$ years old $)$ participated in an 8-session of HIIT supervised through online video calls for two weeks. They were evaluated pre- and post-HIIT for 3-min of burpees, 1-min of sit-ups, maximum push-ups, and 1-min of squats. Perceptions related to training protocols were collected through the Physical Activity Enjoyment Scale (PACES) post-intervention. Results: Significant improvements in burpees (pre:54.15 \pm 18.03 and after: $63.15 \pm 15.18$ ), sit-ups (pre:24.38 \pm 13.38 and after: $31.15 \pm 13.15$ ), push-ups (pre: $9.46 \pm 6.59$ and after: $13.85 \pm 7.33$ ) and squats (pre: $48.00 \pm 8.37$ and after: $54.85 \pm 8.79$ ) were observed. PACES revealed positive perceptions related to the enjoyment of participating in this HIIT. Conclusion: Virtually supervised HIIT improved physical performance in young female athletes during the COVID-19 pandemic, and maybe an efficient strategy for the quarantine period.
\end{abstract}

Keywords: adolescent, high-intensity interval training, athletes, internet-based intervention and women.

\section{Introduction}

Due to the context of the global pandemic caused by Coronavirus Disease 2019 (COVID-19), athletes of team sports had their training and competitions canceled or postponed during the year $2020^{1}$. Thus, athletes are finding challenges to mitigate the loss of physical conditioning, strength, power, and muscle mass during social isolation $^{2}$. In order to minimize the consequences of the COVID-19 quarantine related to athletes' physical fitness, strategies to organize guidance to multidisciplinary support, and to plan a strength and conditioning training at home with adequate space and accessible materials have been recommended ${ }^{3}$.

High-intensity interval training (HIIT) is an efficient method to improve aerobic and anaerobic performance in adolescents ${ }^{4}$. Previously, a study showed that 12 weeks of three 12-min HIIT sessions per week improve muscle strength and cardiovascular fitness in teenagers ${ }^{5}$. However, researchers also identified increases in metabolic and cardiovascular responses in short interventions, as 2-week of HIIT $^{6,7}$. Additionally, calisthenics HIIT protocols can improve physical fitness ${ }^{8}$, and it seems to be an effective strategy for home-based training ${ }^{9,10}$. Thus, using homebased HIIT protocols in team-sport young athletes can mitigate losses or maintain their physical fitness during the COVID-19 quarantine.

In recreationally active females, 4-week of HIIT program with low-volume and whole-body improved aerobic capacity and muscular endurance ${ }^{11}$. However, the literature is limited, considering the HIIT effects on neuromuscular responses in females, young athletes, and strategies to team-sport athletes keeping physically active during social isolation are being needed. Therefore, this study aimed to investigate the effects of a 2-week HIIT intervention on physical performance through online supervised training during COVID-19 quarantine in female young rugby athletes.

\section{Material and methods}

\section{Participants}

Thirteen rugby athletes $(15.92 \pm 0.76$ years old) participated in this study. The participants were part of a longterm athlete development project; however, they had not participated in a systematic training program for at least two months due to the Coronavirus pandemic. Athletes who did not complete the eight training sessions nor preand post-intervention evaluations or were injured during 
the intervention period were excluded from this study. The project in which the athletes participate collected the signature of the consent form of the legal guardians and the consent form of the athletes to carry out the investigation and the project was approved by the local Ethics Committee under the number $\# 2.243 .675$.

\section{Study design}

This intervention study investigated two-week highintensity interval training (HIIT) at home supervised by video calls on the physical performance of young female athletes. The intervention consisted of eight HIIT sessions with two different protocols of approximately $30 \mathrm{~min}$, performed four times per week. The pre and post-tests were carried out to investigate the effects of the intervention on physical performance. Figure 1 shows the study design.

\section{Procedures}

All evaluations and training sessions were performed through recorded video calls. The video call was made and recorded through the Zoom Video Communications platform (Version 2020, Eric Yuan, San Jose, USA). Therefore, the application of the tests required a stable internet connection between evaluator and athlete, individually. When the connection did not allow counting the repetitions during the evaluation, the test was repeated another day. The tests were conducted by an evaluator during the video call with a presence of an assistant previously instructed to count the movements. Before the tests, the participants performed a standardized warm-up composed of three sets of 15 air squats, 10 hip mobility, front plank during $45 \mathrm{~s}$, and three sets of $45 \mathrm{~s}$ of lowintensity skipping, and $15 \mathrm{~s}$ high-intensity skipping. Furthermore, 5 min of rest were respected between each test. The symptoms related to the menstrual period were controlled for physical tests. Therefore, evaluations were conducted when reported absence of symptoms related to the menstrual cycle.

Considering the peak height velocity (PHV) of girls occurs at approximately 11 years of age ${ }^{12,13}$, changes in physical performance due to biological maturation would be mitigated up to 3 years after $\mathrm{PHV}^{14}$. Therefore, this study did not assess the biological maturation of individuals.

\section{Physical performance tests}

Burpee Test: The 3-min burpee test consisted of performing as many burpees as possible for three minutes ${ }^{15}$. The valid burpee consisted of the following sequence: placing the hands on the floor, moving the feet back to the hip extension, returning with the feet in the starting position, extending the hips and knees, and joining the palms above the head ${ }^{14}$. The test was recorded, and later the burpees were counted and registered.

Sit-up Test: The 1-min sit-up test consisted of performing as many sit-ups as possible in one minute. The performance position was in the supine position, with elbows bent, hands crossed in front of the chest, knees bent, and with the aid of an assistant, the athlete kept her feet fixed on the ground. The valid abdominal movement was to perform hip flexion until the elbows are in contact with the knees and return to the initial position ${ }^{16}$. The count was checked and recorded in the video recording.

Push-up Test: The push-up test consisted of maintaining a horizontal position with feet and hands on the floor and performing a complete flexion movement of approximately $90^{\circ}$ followed by elbow extension without intervals greater than 2 seconds between repetitions. The test was completed when the athlete gave up or was unable to complete the movement ${ }^{17}$. The count of the number of complete repetitions was recorded.

Squat Test: The test consisted of performing as many squats as possible for one minute. The squat's full valid movement was to start from the neutral position, perform knee flexion at $90^{\circ}$, and return to the neutral position ${ }^{18}$. The counted number of valid repetitions was checked in the video recording.

\section{Intervention}

A 10-min warm-up and 10-min cool-down routines were conducted before and after the HIIT protocols. The warm-up consisted of three sets of 15 air squats, 10 hip mobility, front plank during $45 \mathrm{~s}$, and three sets of $45 \mathrm{~s}$ of low-intensity skipping, and $15 \mathrm{~s}$ high-intensity skipping. The cool-down consisted of two sets of $30 \mathrm{~s}$ of hamstring,

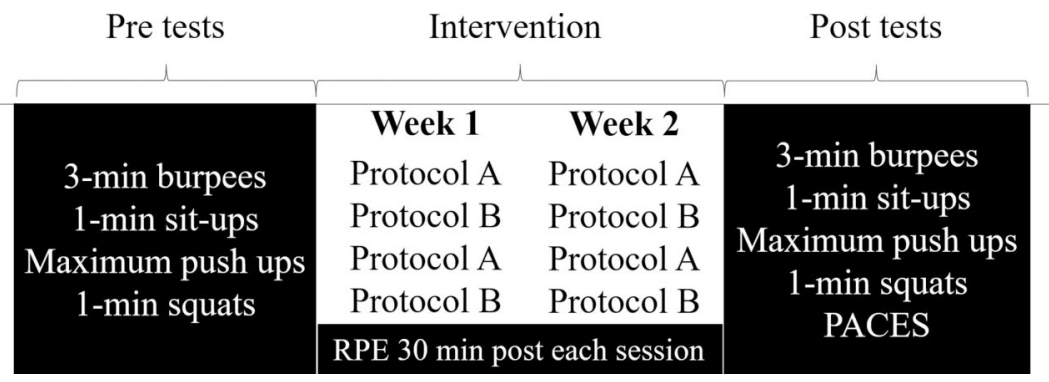

Figure 1 - Study design. Legend: RPE = Rating of Perceived Exertion (Borg's scale). PACES = Physical Activity Enjoyment Scale. 
abdomen, chest, and back stretching exercises. The HIIT consisted of 8 sessions of 2 different protocols presented in Figure 2. Briefly, the protocols consisted of making efforts of $20 \mathrm{~s}$ (protocol A) and $60 \mathrm{~s}$ (protocol B) interspersed with the passive recovery of $10 \mathrm{~s}$ (protocol A) and $60 \mathrm{~s}$ (protocol $\mathrm{B}$ ). In protocol $\mathrm{A}$, two cycles of 8 series were performed, separated by $4 \mathrm{~min}$, and in protocol $\mathrm{B}$, a cycle of 6 series was conducted. The protocols' construction was based on the McRae et al. ${ }^{11}$ and Scott et al. ${ }^{9}$ studies. During the intervention, the 0-10 Borg's rating of perceived exertion (RPE) scale for each session was collected $^{19}$. To this, the $0-10$ scale was registered 30 min after the session.

Additionally, perceptions related to training protocols were collected through the Physical Activity Enjoyment Scale (PACES) post-intervention sent by Google Forms ${ }^{\circledR}$ link. The PACES is a 16-question questionnaire comprising a 5-point scale validated for young people ${ }^{20}$. The sum of the scoring items calculated the PACES score. To this, seven items were inverted as to the positive response (I feel bored; I dislike it; It's no fun at all; It makes me sad; It frustrates me; It's not at all interesting; I feel as though I would rather be doing something else). Therefore, the score could vary between 16 and 80 points.

\section{Data analysis}

The Shapiro Wilk test confirmed the normality of the physical fitness test data, and these were presented as mean and standard deviation. A paired $t$-test to identify differences in performance tests between pre- and postintervention, ANOVA with repeated measures to identify
RPE differences among sessions, and median (from the minimum score to maximum score) and frequencies on the PACES scale were conducted.

\section{Results}

Table 1 shows significant improvements post-intervention compared to pre-intervention in all the tests $(\mathrm{p}<0.01)$. Also, there are no differences in RPE among sessions, as shown in Figure $3(\mathrm{p}=0.181)$. The variation delta of the pre- and post-intervention tests was burpee $=$ $20.05 \pm 16.91 \%$; sit-up $=40.25 \pm 50.13 \%$; push-up $=$ $100.71 \pm 170.92 \%$; squat $=15.44 \pm 17.35 \%$. In addition, PACES revealed a score median of 70 points (from 59 to 76 points) related to the enjoyment of participating in the HIIT.

\section{Discussion}

This study investigated the effects of a 2-week home-based HIIT on physical performance through online supervised training during COVID-19 quarantine. The main results showed improvements in burpee, sit-up, push-up, and squat tests. Also, the RPE did not show differences during the sessions, and PACES showed positive perceptions related to the enjoyment during the intervention. It highlighted the practical relevance as the strength of this study, which showed that, given the pandemic context, home-based HIIT methods could contribute to young female athletes' physical fitness.

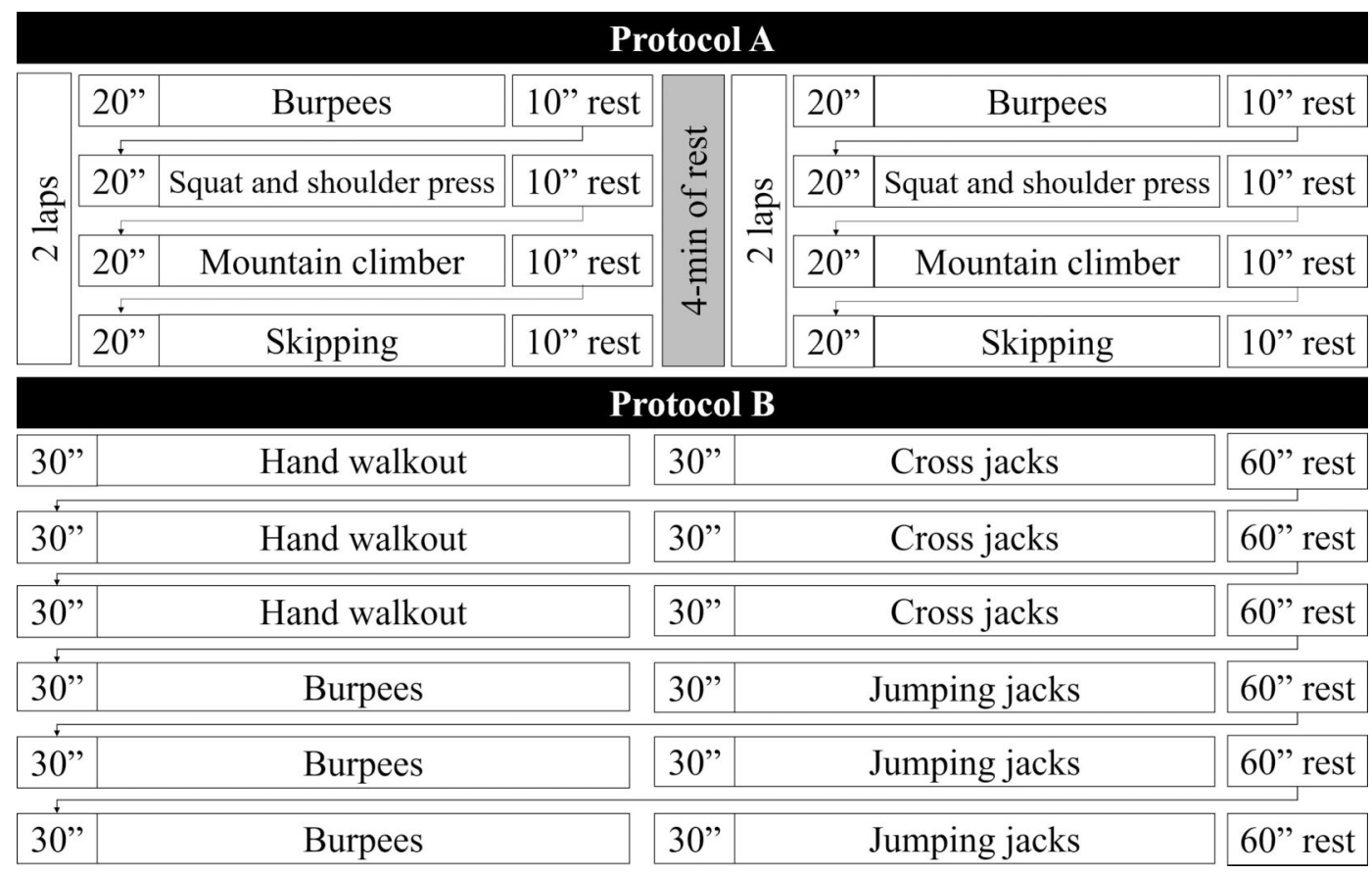

Figure 2 - Training protocols. 
Table 1 - Mean and standard deviation of pre and post-intervention tests $(n=13)$.

\begin{tabular}{|c|c|c|c|c|c|}
\hline \multirow[t]{2}{*}{ Test } & \multicolumn{2}{|c|}{ Mean \pm SD } & \multirow[b]{2}{*}{ p-value ${ }^{a}$} & \multirow[b]{2}{*}{ Effect size } & \multirow[b]{2}{*}{ Magnitude } \\
\hline & Pre & Post & & & \\
\hline Burpee, rep & $54.15 \pm 18.03$ & $63.15 \pm 15.18^{\mathrm{b}}$ & 0.001 & 0.50 & Small \\
\hline Sit-up, rep & $24.38 \pm 13.38$ & $31.15 \pm 13.15^{\mathrm{b}}$ & 0.002 & 0.51 & Small \\
\hline Push-up, rep & $9.46 \pm 6.59$ & $13.85 \pm 7.33^{\mathrm{b}}$ & 0.001 & 0.67 & Small \\
\hline Squat, rep & $48.00 \pm 8.37$ & $54.85 \pm 8.79^{\mathrm{b}}$ & 0.004 & 0.82 & Moderate \\
\hline
\end{tabular}

${ }^{\mathrm{a}}$ Calculated from T-test comparing the average of pre and post-intervention values in each test. ${ }^{\mathrm{b}}$ Significantly different from pre-intervention $(\mathrm{p}<0.01)$.

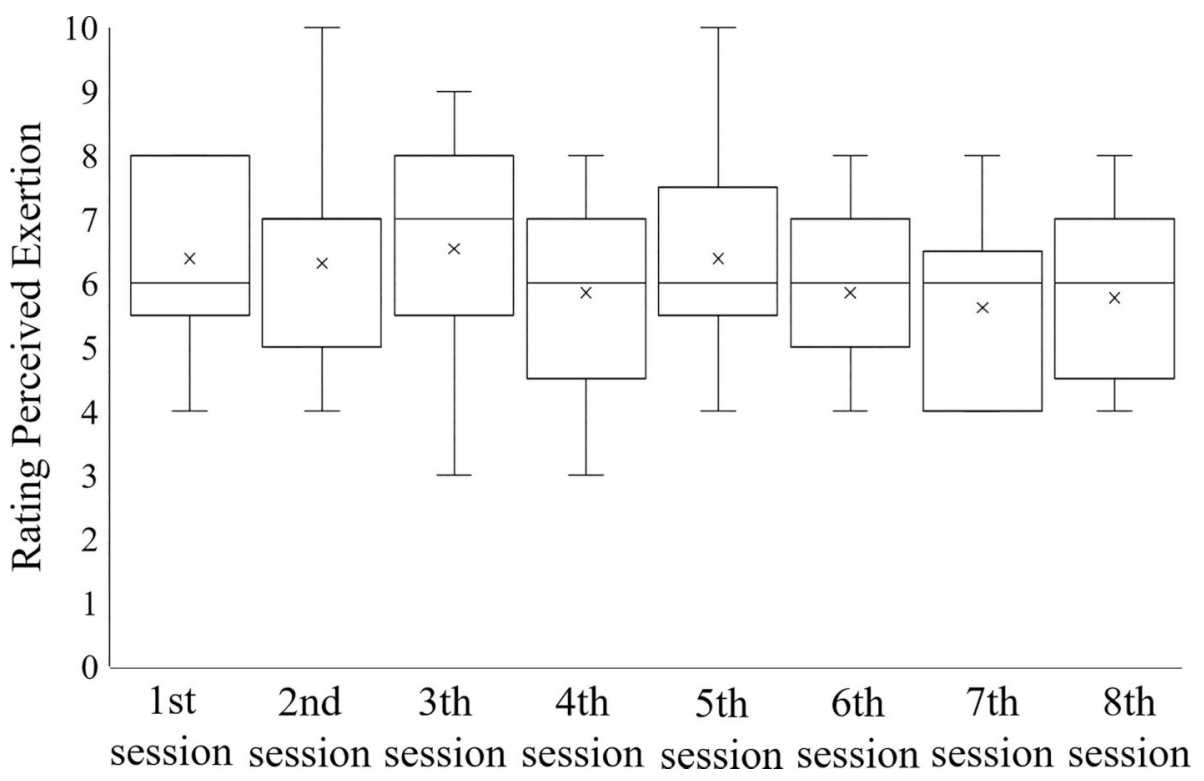

Figure 3 - Rating Perceived Exertion 30-min post each training session.

Young women (age $=20.4 \pm .6$ years) carried out a 2-week HIIT intervention with ten cycle ergometer sessions ${ }^{7}$. They were compared to the control group (age $=21.6 \pm 0.4$ years) for anaerobic power and metabolic and cardiorespiratory responses. The intervention group significantly improved peak power (from $492.1 \pm$ 19.4 to $617.2 \pm 13.6 \mathrm{~W}$ ) and mean power (from $351.1 \pm$ 8.2 to $377.5 \pm 9.6 \mathrm{~W}$ ) in the Wingate test, maximum oxygen consumption $\left(\mathrm{VO}_{2 \max }\right.$; from $34.27 \pm 0.71$ to $37.38 \pm$ $\left.1.02 \mathrm{~mL} \cdot \mathrm{kg}^{-1} \cdot \mathrm{min}^{-1}\right)$, maximal carbon dioxide production $\left(\mathrm{VCO}_{2 \max }\right.$; from $2208.25 \pm 79.76$ to $2441.36 \pm$ $34.42 \mathrm{~mL} \cdot \mathrm{min}^{-1}$ ), ventilatory equivalent (from $82.26 \pm$ 7.03 to $98.98 \pm 7.21 \mathrm{~L} \cdot \mathrm{min}^{-1}$ ) and carbohydrate oxidation (from $3.48 \pm 0.16$ to $3.91 \pm 0.12 \mathrm{~g} \cdot \mathrm{min}^{-1}$ ) in an incremental test. In contrast, the control group did not find differences between moments ${ }^{7}$. Active young men and women $(n=20$, age $=25.3 \pm 4.5$ years $)$ performed a 2 week HIIT intervention of 6 sessions using a cycle ergometer ${ }^{6}$. Compared to the control group $(\mathrm{n}=9$, age $=22.8 \pm 2.8$ years), results revealed significant improvements in power output, $\mathrm{VO}_{2 \max }$ (from $43.6 \pm 5.4$ to $\left.46.0 \pm 5.2 \mathrm{~mL} \cdot \mathrm{kg}^{-1} \cdot \mathrm{min}^{-1}\right), \mathrm{VCO}_{2 \max }(3.7 \pm 0.8$ to 3.9 $\left.\pm 0.9 \mathrm{~L} \cdot \mathrm{min}^{-1}\right)$, and oxygen pulse $(16.9 \pm 4.3$ to $17.9 \pm$
$4.5 \mathrm{~mL} \cdot$ beat $^{-1}$ ) with interactions group and moment ${ }^{6}$. The present study applied a 2 -week intervention with eight HIIT sessions and identified improvements in the physical tests, corroborating with previous studies. In this sense, two weeks of HIIT seems to provide positive metabolic and performance responses in young females.

Although HIIT with Wingate-type exercise is efficient in metabolic and cardiorespiratory responses, different HIIT models are more feasible to improve physical fitness $^{21}$. An investigation observed the effects of a 2week HIIT program using calisthenics exercise (burpees) in inactive male and female students $(\mathrm{n}=23$; age $=22.08$ \pm 0.99 years). This result identified improvements in $\mathrm{VO}_{2 \max }$ (from $34.44 \pm 8.88$ to $38.52 \pm$ $52.00 \mathrm{~mL} \cdot \mathrm{kg}^{-1} \cdot \mathrm{min}^{-1}$ ), shuttle run test (SRT; from 940.00 \pm 498.57 to $1176.66 \pm 568.75 \mathrm{~m}$ ), heart rate after SRT (from $174.91 \pm 34.27$ to $169.83 \pm 30.93 \mathrm{bpm}$ ), lower limbs strength (from $85.66 \pm 27.58$ to $94.54 \pm 30.00 \mathrm{~kg}$ ) and back strength (from $83.95 \pm 26.18$ to $98.70 \pm$ $25.83 \mathrm{~kg}$ ), with no differences in the control group ${ }^{8}$. In the present study, the HIIT protocols also were composed of calisthenic exercises, performed in as many repetitions as possible in the given time. As in the previous study ${ }^{8}$, the 
present investigation identified improvements in physical performance in females non-accustomed to HIIT, regarding the increase in the number of repetitions before and after the intervention (burpee $=54.15 \pm 18.03$ to $63.15 \pm$ 15.18 repetitions, sit-up $=24.38 \pm 13.38$ to $31.15 \pm 13.15$ repetitions, push-up $=9.46 \pm 6.59$ to $13.85 \pm 7.33$ repetitions, and squat $=48.00 \pm 8.37$ to $54.85 \pm 8.79$ repetitions). Another investigation observed that 4-week HIIT intervention using calisthenics exercises (burpees, jumping jacks, mountain climbers, or squat thrusts) in female students $(\mathrm{n}=7$; age $=20.7 \pm 1.0$ years $)$, and improvements in $\mathrm{VO}_{2 \text { peak }}(\sim 8 \% ; \mathrm{p}<0.05)$ and muscle endurance (leg extensions, $+40 \%$; chest presses, $+207 \%$; sit-ups, $+64 \%$; push-ups, $+135 \%$; and back extensions, $+75 \%$; $\mathrm{p}<0.05$ ) in the HIIT group ${ }^{11}$. In the present study, an increase in the number of $16 \%$ repetitions in burpees, $27 \%$ in sit-ups, $46 \%$ in push-ups, and $14 \%$ in squats were found. Regarding the longer training period and non-athlete subjects in the previous study, the present research results also demonstrated that HIIT protocols at home supervised virtually are efficient in improving neuromuscular resistance.

A study comparing HIIT and moderate-intensity continuous training (MICT) in recreationally active men and women $(\mathrm{n}=12 ; 25.5 \pm 10.7$ years old $)$, using the PACES scale indicated greater enjoyment in HIIT $(p=0.013)$, despite the higher RPE values in $\mathrm{HIIT}^{22}$. In the current investigation, the RPE was similar during the intervention, and the PACES identified positive perceptions in female, young athletes about the HIIT in social isolation. Studies have shown that HIIT protocols with good stimuli-recovery relationships may contribute to positive affective responses in healthy and unhealthy individuals $^{23}$. Therefore, different home-based HIIT protocols may be a strategy to keep young female athletes active with physically efficient and enjoyable training ${ }^{24}$.

As the main limitation, the absence of intensity control measured by internal markers is highlighted. However, seeking to mitigate this bias, the researchers carried out the monitoring of the session load through the RPE, which is an instrument widely used to control the intensity in HIIT protocols applied to the athletic routine $e^{25-27}$ and we maintained verbal stimuli for motivating purposes during the effort. The absence of a control group is another limitation of this study. However, there was control of the following factors: the athletes participated in the same training program up to two months before the intervention; the athletes did not perform any systematic training in the last 2 months before the intervention, and; the athletes did not perform other physical activities during the intervention. Therefore, the results of this study showed that high-intensity interval training had a positive effect on the physical fitness of young athletes.

At the end of the present study, it is possible to highlight significant findings. Firstly, this manuscript supports the scientific literature that indicates HIIT, performed with whole-body exercises using only the body mass, a training method with satisfactory neuromuscular improvements, and easy to apply. Also, for conditioning trainers, the results presented a systematic training protocol, useful for inducing positive responses, applied in a virtual way, which may be opportune considering the current pandemic and, also, this is a strategy of enabling athletes, who for some reason they need to stay away from the general group, physical activities that make their athletic development possible.

\section{Conclusion}

This investigation concluded that a 2-week homebased HIIT intervention performed with the whole-body increased the number of repetitions in burpee, sit-up, push-up, and squat tests, demonstrating that it is efficient to induce positive responses in the endurance strength in female young rugby athletes. In addition, the RPE showed similar values during the intervention, and the PACES identified positive perceptions of enjoyment in practice HIIT. These results may have implications for maintaining training during COVID-19 quarantine for young teamsport athletes.

\section{References}

1. Toresdahl BG, Asif IM. Coronavirus disease 2019 (COVID19): considerations for the competitive athlete. Sports Health. 2020;12(3):221-4. doi

2. Latella C, Haff, GG. Global challenges of being a strength athlete during a pandemic: impacts and sports-specific training considerations and recommendations. Sports. 2020;8(100):1-16. doi

3. Jukic I, Calleja-González J, Cos F, Cuzzolin F, Olmo J, Terrados $\mathrm{N}$, et al. Strategies and solutions for team sports athletes in isolation due to COVID-19. Sports. 2020;8(56):2-9. doi

4. Engel FA, Ackermann A, Chtourou H, Sperlich B. Highintensity interval training performed by young athletes: a systematic review and meta-analysis. Front Physiol. 2018;9 (1012):1-18. doi

5. Abarzúa VJ, Viloff CW, Bahamondes VJ, Olivera Y, Poblete-Aro C, Herrera-Valenzuela T, et al. High intensity interval training in teenagers. Rev Med Chil. 2019;147(2):22130. doi

6. Astorino TA, Allen RP, Roberson DW, Jurancich M. Effect of high-intensity interval training on cardiovascular function, VO2max, and muscular force. J Strength Cond Res. 2012;26(1):138-45. doi

7. Aslankeser Z, Balci \&̈S. Substrate oxidation during incremental exercise in young women: the effects of 2-week high-intensity interval training. Med Sport. 2017;70(2):13749. doi

8. Alsairawan A, Gürpinar B, İlçi?n N. Is 2-week calisthenics high-intensity interval training enough to change aerobic and anaerobic capacity? J Exerc Rehabil. 2019;6(1):25-31. 
9. Scott SN, Shepherd SO, Strauss JA, Wagenmakers AJM, Cocks M. Home-based high-intensity interval training reduces barriers to exercise in people with type 1 diabetes. Exp Physiol. 2020;105(4):571-8. doi

10. Krawcyk RS, Vinther A, Petersen NC, Faber J, Iversen HK, Christensen T, et al. Effect of home-based high-intensity interval training in patients with lacunar stroke: a randomized controlled trial. Front Neurol. 2019;10(664):1-15. doi

11. McRae G, Payne A, Zelt JGE, Scribbans TD, Jung ME, Little JP, et al. Extremely low volume, whole-body aerobicresistance training improves aerobic fitness and muscular endurance in females. Appl Physiol Nutr Metab. 2012;37 (6):1124-31. doi

12. Bergmann GG, Bergmann ML, Lorenzi T, Pinheiro EP, Garlipp DC, Moreira RB, et al. Peak growth velocity of height, body mass and subcutaneous fat in 10 to 14-year-old boys and girls. Rev Bras Cineantropom Desempenho Hum. 2007;9(4):333-8.

13. Malina RM, Rogol AD, Cumming SP, Coelho e Silva MJ, Figueiredo AJ. Biological maturation of youth athletes: assessment and implications. Br J Sports Med. 2015;49 (13):852-9. doi

14. Hebestreit H, Bar-Or B, editors. The young athlete. London, International Olympic Committee; 2008.

15. Podstawski R, Markowski P, Clark CCT, Choszcz D, Ilhász F, Stojiljkovic S, Gronek P. International standards for the 3min burpee test: high-intensity motor performance. J Hum Kinet. 2019;69(1):137-47. doi

16. Simon JE, Docherty CL. The impact of previous athletic experience on current physical fitness in former collegiate athletes and noncollegiate athletes. Sports Health. 2017;9 (5):462-8. doi

17. Hashim A. Objectivity, reliability, and validity of the $90^{\circ}$ push-ups test protocol among male and female students of sports science program. J Phys Educ. 2012;12(1):103-6. doi

18. Pereira MIR, Chagas PS. Muscle strength and endurance tests: reliability and prediction of a maximum repetitionreview and new evidence. Rev Bras Med Esporte. 2003;9 (5):325-35. doi

19. Shariat A, Cleland JA, Danaee M, Alizadeh R, Sangelaji B, Kargarfard M, et al. Borg CR-10 scale as a new approach to monitoring office exercise training. Work. 2018;60(4):54954. doi

20. Moore JB, Yin Z, Hanes J, Duda J, Gutin B, Barbeau P. Measuring enjoyment of physical activity in children: validation of the physical activity enjoyment scale. J Appl Sport Psychol. 2009;21(1):116-29. doi
21. Gillen JB, Gibala MJ. Is high-intensity interval training a time-efficient exercise strategy to improve health and fitness? Appl Physiol Nutr Metab. 2014;39(3):409-12. doi

22. Thum JS, Parsons G, Whittle T, Astorino TA. High-intensity interval training elicits higher enjoyment than moderateintensity continuous exercise. Plos One. 2017;12(1): e0166299. doi

23. Oliveira BRR, Santos TM, Kilpatrick M, Pires FO, Deslandes AC. Affective and enjoyment responses in high-intensity interval training and continuous training: A systematic review and meta-analysis. Plos One. 2018;13(6):e0197124. doi

24. Nasci ABA, Orcy RB, Cabistany LD, Formalioni A, Del Vecchio FB. Acute responses of high-intensity circuit training in women: Low physical fitness levels show higher muscle damage. Rev Bras Cineantropometria Desempenho Hum. 2018;20(5):391-401. doi

25. Costa JA, Brito J, Nakamura FY, Figueiredo P, Rebelo A. Using the rating of perceived exertion and heart rate to quantify training intensity in female soccer players: validity and utility. J Strength Cond Res. 2019;1:1-6 [Online ahead of print]. doi

26. Tibana RA, Sousa NMF, Cunha GV, Prestes J, Fett C, Gabbett TJ, Voltarelli FA. Validity of session rating perceived exertion method for quantifying internal training load during high-intensity functional training. Sports (Basel). 2018;6(68):1-8. doi

27. Scott SN, Shepherd SO, Hopkins N, Dawson EA, Strauss JA, Wright DJ, et al. Home-hit improves muscle capillarisation and eNOS/NAD(P) hoxidase protein ratio in obese individuals with elevated cardiovascular disease risk. J Physiol. 2019;597(16):4203-25. doi

\section{Corresponding author}

Camila Borges Müller. Universidade Federal de Pelotas, Escola Superior de Educação Física, Pelotas, RS, Brazil. E-mail: camilaborges1210@gmail.com.

Manuscript received on July 27, 2021

Manuscript accepted on September 1, 2021

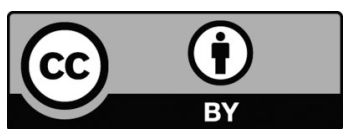

Motriz. The Journal of Physical Education. UNESP. Rio Claro, SP, Brazil - eISSN: 1980-6574 - under a license Creative Commons - Version 4.0 